\title{
Special section: Advances in artificial intelligence in biomedical image analysis
}

๑) Australasian College of Physical Scientists and Engineers in Medicine 2018

Special section: Articles expressly submitted by authors that address an issue that is pertinent to:

Advances in Artificial Intelligence in Biomedical Image Analysis (Some articles that were submitted for the special section did not complete the peer review process in time to be included. They will appear in the March 2019 issue).

\section{Guest Editors:}

Kelvin Wong ${ }^{1}$, Jianxu Chen ${ }^{2}$

${ }^{1}$ School of Medicine, Western Sydney University, Sydney, Australia

${ }^{2}$ Allen Institute for Cell Science, USA.
The aim of this Special Section is to source and publish high quality articles that involve Artificial Intelligence in Biomedical Image Analysis. They fall within the area of interest of the Journal and/or the boundaries of the Special Section as they have a AI application.

(All articles were subject to final review and acceptance by the APESM Editor in Chief.) 